\title{
Role of Paediatric Dentist in Non-Invasive Management of Obstructive Sleep Apnoea in Children
}

\author{
Megha Sethi ${ }^{1}$, Shveta Sood ${ }^{2}$, Naresh Sharma ${ }^{3}$, Akshara Singh ${ }^{4}$ \\ 1, 2, 3, 4 Department of Pedodontics and Preventive Dentistry, \\ Manav Rachna Dental College, MRIIRS, Faridabad, Haryana, India.
}

\section{ABSTRACT}

\section{BACKGROUND}

Obstructive sleep apnoea (OSA) is a chronic disorder in which repetitive episodes of cessation of breathing occurs during sleep due to collapse of upper airway leading to recurrent awakening, sleep fragmentation, and perturbation in ventilatory function. The disorder is much more common than realised and the manifestations in children are different from those in adults. OSAS may present in different ways like cognitive dysfunction, excessive day time somnolence, decreased attention span, drooling of saliva, mood disorders like depression or irritability as well as adverse cardiovascular sequelae like hypertension, heart failure. Polysomnography or overnight sleep studies remain the gold standard for the diagnosis of paediatric obstructive sleep apnoea (POSA) disorder. Therapeutic approaches for POSA include surgical and nonsurgical means. The choice of therapy depends on various factors like aetiology, severity of the POSA and patient's choice. Although continuous positive airway pressure (CPAP) remains the first line therapy in adults, the introduction of oral appliances provided an attractive alternative and made the role of dentists more prominent in management of this disorder. OSA is often under-diagnosed due to lack of awareness. So, it is essential for dentists to be aware of the various aspects of this disorder as well as its management.

\section{KEY WORDS}

OSA, Paediatric Dentist, Oral Appliances, Non-Invasive Management
Corresponding Author: Dr. Megha Sethi, Department of Pedodontics and Preventive Dentistry, Manav Rachna Dental College, Faculty of Dental Sciences, MRIIRS, Sector-43, Delhi- Surajkund Badhkal Road, Faridabad - 121004, Haryana, India. E-mail: docmeghs@gmail.com

DOI: $10.14260 / j e m d s / 2021 / 266$

How to Cite This Article:

Sethi M Sood S, Sharma N, et al. Role of paediatric dentist in non-invasive management of obstructive sleep apnoea in children. J Evolution Med Dent Sci 2021;10(17):1251-1256, DOI: 10.14260/jemds/2021/266

Submission 24-12-2020,

Peer Review 26-02-2021

Acceptance 03-03-2021,

Published 26-04-2021.

Copyright (c) 2021 Megha Sethi et al. This is an open access article distributed under Creative Commons Attribution License [Attribution 4.0 International (CC BY 4.0)] 


\section{BACKGROUND}

The term sleep disordered breathing (SDB) is used to describe the breathing disorders occurring during sleep. It includes habitual snoring, upper airway resistance syndrome (UARS) as well as obstructive sleep apnoea (OSA). ${ }^{1}$

Sleep apnoea can be classified as central, obstructive, or mixed. In central sleep apnoea (CSA) the hypoventilation is caused due to decreased respiratory drive during sleep. In central sleep apnoea, there is an inadequacy of respiratory effort during cessations of airflow, whereas in OSA, breathing efforts are present. OSA is likely the most prevalent of all the apnoea's. The term obstructive sleep apnoea syndrome (OSAS) is used when these apnoeic-hypopnoeic episodes are associated with symptoms like excessive day time sleepiness. A patient suffering from mixed apnoea show characteristics of both CSA and OSA. ${ }^{2}$

In the beginning, the diagnostic criteria used to diagnose OSA in children was same as that used in adults. As it was progressively realised that the neurological development continues throughout the childhood, and that symptoms and polysomnographic features of OSA in childhood are quite different from those of adults, changes were made in the evaluation of paediatric SDB and positional obstructive sleep apnoea (POSA). ${ }^{3}$

Symptoms of POSA include loud snoring for $>3$ nights per week, witnessed episodes of breathing cessation, sudden arousal along with shortness of breath, symptoms of mouth breathing like dry mouth or sore throat, unrefreshing sleep, and also, frequent morning headaches. These symptoms may lead to neurobehavioral consequences like hyperactivity, inattention, irritability, resulting in growth disturbances and poor academic performance. ${ }^{3}$ Daytime consequences of SDB may affect neurobehavioral, cardiovascular, and / or inflammatory systems. ${ }^{4}$

The management of OSA requires multi-disciplinary approach including general physician, sleep medicine physician, dentists, dieticians and others. The dentists have a central role in the management of sleep disorders, which goes beyond the identification and referral for evaluation, to treatment using various oral appliances. So, it is of utmost importance for dentists to be familiar with various aspects of OSA.

\section{EPIDEMIOLOGY}

The prevalence of SDB in children was about $12 \%$ to $15 \%{ }^{5}$ The highest prevalence was found between 3 and 5 years of age. ${ }^{5}$ Habitual snoring is defined when it is present for more than 3 nights / week, and was seen in $3-12 \%$ of children. ${ }^{6}$ Most of the studies report prevalence of POSA to range from 1 - $5 \%$ of all children. ${ }^{6}$ OSA in many children may remain undiagnosed as the presentation may be quite different in children compared to grown-ups. ${ }^{3}$ In contrast to adults, both genders are affected equally. ${ }^{7-9}$ However, male predominance is seen post puberty. ${ }^{10}$

\section{PATHOPHYSIOLOGY}

Obstructive sleep apnoea is a chronic disease in which repetitive episodes of cessation of breathing occur during sleep due to collapse of upper airway. This leads to recurrent awakening, sleep fragmentation, and perturbation in ventilatory function. ${ }^{3}$ The pathophysiology of OSA involves a complex interaction between narrowed upper respiratory tract which makes it prone to collapse, and effect of sleep on neural control of respiratory system. During the wakeful state, the narrowed upper airway is prevented from collapsing by compensatory neuronal activation of dilator muscles, which is lost during sleep and the airway collapses partially or completely.

The role of obesity in pathophysiology of POSA is multifaceted, as it results in decreased caliber of the upper airway due to fat deposition in upper airway structures, as well as, increase in resistive load of the respiratory system, due to excess adipose tissue deposition around the thoracic and abdominal walls, decreasing diaphragm excursion and tidal volume, particularly during supine posture during sleep. ${ }^{11}$ Also, pro-inflammatory milieu is seen in obesity as well as POSA. The interaction between obesity and POSA is intricate, as obesity can predispose to POSA, whereas POSA can itself serve as a risk factor for obesity. ${ }^{12}$

\section{AETIOLOGY}

The most common risk factors for SDB in paediatric age group are adenotonsillar hypertrophy followed by obesity, and craniofacial features like choanal atresia, micrognathia, narrow palatal arch, dolichocephaly, high arched palate, deviated nasal septum, macroglossia, and retrognathia, steeper and shorter anterior cranial base, inferiorly displaced hyoid bone. $3,4,13$

Obesity is a major risk factor in the pathophysiology of OSA in adults. As the prevalence of obesity is increasing in childhood, a concomitant rise in prevalence of POSA is also seen. ${ }^{14} \mathrm{~A}$ study reported increase in risk of POSA by $12 \%$ as the body mass index (BMI) increases by $1 \mathrm{~kg} / \mathrm{m}^{2,7}$ Dayyat et al. reported that the degree of adenotonsillar hypertrophy required for POSA to manifest, is less in obese children. ${ }^{15}$

Premature birth has also been associated with higher risk of developing SDB during childhood, likely due to disturbances like hypotonia, altered growth of orofacial structures, as well as mouth breathing. ${ }^{4}$ The degree of hypotonicity is directly related to the degree of prematurity in the infant. ${ }^{4}$

The enlargement of the soft tissues leading to narrowing of the upper airway can be caused by systemic diseases like amyloidosis, hypothyroidism, acromegaly, and nutritional deficiencies or metabolic disorders such as polysaccharidosis.13 Gastroesophageal reflux can lead to oedema of the upper airway in infants which can predispose to the development of OSA. ${ }^{3}$ Narrowing of the airway can also be caused by respiratory tract allergies like asthma. ${ }^{13}$ The alterations in cranio-facial anatomy is influenced by genetic conditions like Achondroplasia, Treacher Collins syndrome, which predisposes patients to the development of OSA. ${ }^{13}$

Dental agenesis may lead to oral breathing as well as decrease in the width of dental arches, which may result in 
SDB. ${ }^{16}$ Sleep bruxism was reported in up to $50 \%$ of children suffering from POSA. ${ }^{17}$ As the dentists commonly encounter this condition in their practices, they can recognize such children and counsel their parents about the increased risk for POSA and refer them for further evaluation and treatment.

\section{CLINICAL MANIFESTATIONS}

OSAS may present in different ways like cognitive dysfunction, excessive day time somnolence, decreased attention span, drooling of saliva, mood disorders like depression or irritability, dry mouth, and witnessed apnoeic episodes (Table 1). These symptoms eventually lead to poor quality of life which is reflected in poor performance at workplace, impaired social interactions and may even sometimes lead to life threatening situations like road traffic accidents. ${ }^{18}$ The risk of road traffic accidents in drivers with untreated OSAS, was reported to be two or three times higher compared to those in matched control group. ${ }^{19}$ Some studies have reported worsening of conditions like epilepsy, asthma, and hypertension in patients who are not on any treatment for OSA.

\begin{tabular}{|cc|}
\hline Symptoms & Signs \\
\hline Snoring & Increased body weight \\
Excessive daytime somnolence & Enlarged neck circumference \\
Choking or gasping at night & Narrow upper airway \\
Night sweats & Hypertension \\
Cognitive disorders & Accentuated P2 heart sounds (pulmonary HTN) \\
Dyspepsia & Retrognathia / overjet \\
\hline Morning headaches & Nasal obstruction \\
Insomnia & Decreased oxygen saturation \\
\hline Nocturia & Pedal oedema (heart failure) \\
\hline Table 1. Clinical Manifestations of OSA \\
\hline
\end{tabular}

\section{DIAGNOSIS}

Diagnosis of OSA is suspected in the presence of daytime symptoms like excessive somnolence, poor academic performance. The assessment of sleepiness can be done using the Epworth Sleepiness Scale (ESS), which can help differentiate between expected and excessive daytime sleepiness. ${ }^{20}$ It measures the propensity of the affected person to fall asleep while doing routine activities like watching television, driving, or reading: $0=$ never fall asleep, $1=$ slight chance of falling asleep, 2 = moderate chance of falling asleep, and $3=$ high chance of falling asleep. A score of $>10$ indicates excessive daytime sleepiness and warrants further investigation.

Physical examination may reveal presence of risk factors like obesity, adenotonsillar hypertrophy, cranio-facial abnormalities. Polysomnography, or overnight sleep studies, remain the gold standard for the diagnosis of POSA. During this study, various events and parameters were recorded using electroencephalography (EEG), electrooculography (EOG), electromyography (EMG) and electrocardiography (ECG). Various physiological variables like sleeping posture, breathing pattern, level of oxygen, blood pressure was also recorded.

The apnoea-hypopnea index (AHI), is used to define and assess the severity of POSA. It is defined as the average number of obstructive events per hour of total sleep time. An apnoeahypopnoea index value greater than 1 is considered abnormal in a child. Apnoea is defined as the cessation of airflow for at least $10 \mathrm{sec}$ associated with a simultaneous decrease in arterial oxygen saturation by 2 to $4 \%$ whereas, hypopnea is defined as at least 30 to $50 \%$ reduction in airflow along with desaturation. Table 2 show the severity grading of AHI.

\begin{tabular}{|cc|}
\hline Severity of OSA & AHI \\
Mild & $<5$ \\
Moderate & $>5<10$ \\
Severe & $>10$ \\
\hline \multicolumn{2}{c}{ Table 2. Severity of OSA } \\
\hline
\end{tabular}

Split-night polysomnography offers advantage of diagnosing OSA and assessing the effect of CPAP therapy as well as its titration, in a single night of attended sleep testing. One can also use it to assess the alterations in breathing pattern or frequency of obstructive events at different positions of mandibular advancement device, in patients using an adjustable airway dilator.

The facility of portable sleep studies (PSG and CPAP titration) can be availed by patients who are unable to visit a hospital. Oximetry is a portable and cost-effective means to assess treatment response.

Due to the variation in specific time in seconds is not applicable to children. At least $30 \%$ reduction in airflow along with or without an arousal and with oxygen desaturation of 3 - 4 per cent is more appropriate description of hypopnea.

\section{TREATMENT CONSIDERATIONS}

Therapeutic approaches for POSA include surgical and nonsurgical means. The choice of therapy depends on various factors like aetiology, severity of the POSA and patient's choice. In the present article, the focus will be on the part that a dentist plays in non-invasive strategies for treatment of OSA in children.

\section{NON-SURGICAL APPROACHES}

Nonsurgical therapeutic approaches include positive airway pressure mask, weight loss, pharmacological agents, oral appliance therapy, positional training and myofunctional therapy. Most patients need more than one of these approaches, which requires involvement of multiple disciplines, including general physicians, otolaryngologists, sleep medicine physicians, myofunctional therapists, and dentists.

Pulmonary alveolar proteinosis (PAP)is considered in children in whom adenotonsillar hypertrophy is mild, or symptoms still persist after adenotonsillar resection. The goal of PAP therapy is to keep the airway patent during sleep, thereby reducing the $\mathrm{AHI}$ events and daytime symptoms. It can be delivered either as continuous positive airway pressure (CPAP) or as bilevel pressure (BPAP) application. Although it is very effective in ameliorating the symptoms of POSA, noncompliance is a major hindrance in long term use like inappropriate size and fit of mask, especially for children younger than 2 years of age, as well as craniofacial 
malformations. Compliance can be improved to certain extent by using behavioural therapy including motivational training.

The target of some therapeutic approaches is to modify the risk factors contributing to pathophysiology of OSA. Obesity is a major risk factor of POSA, and therefore, weight reduction is recommended as one of the initial steps.

Certain pharmacological agents like anti-inflammatory drugs including leukotriene receptor antagonists such as montelukast and intranasal steroids can also be used in the treatment of POSA. These agents may be particularly useful in children with respiratory tract allergy like asthma. Intranasal steroids can also be used when adenotonsillar hypertrophy recurs following AT, or when AT cannot be performed. The rationale behind using these agents is to arrest the inflammatory growth of adenotonsillar tissue, thereby, decreasing its size. Although local application of steroids is quite safe as the dose required to produce desired effect is lower than systemic dose. Prolonged use can cause certain adverse effects like nasal irritation, epistaxis and local opportunistic infections like Candidiasis.

\section{NON-SURGICAL DENTAL CONSIDERATIONS}

The introduction of adjustable mandible-advancing oral appliances in clinical practice in 1990, propelled the field of dentistry in active management of OSA, leaving behind the era of mere recognition and referral to sleep physician. Soon, it became the predominant form of dental therapy for OSA.

Oral devices (Table 3), which are thermoplastic materials with retainers and supports, include mandibular repositioning or advancement devices (MRD / MAD). The position can be titrated as well in some of them, e.g., Herbst appliance / snore guard / silencer.

The purpose of these devices is to engage one or both of the dental arches and alter mandible's position. Some novel devices combine an oral appliance with CPAP, which apply positive pressure directly into the oral cavity, thereby eliminating the requirement of head gear or nasal mask. This improves the patient's compliance as well as averts the issue of air leaks.

Tongue Repositioning or Retaining Devices (TRD), e.g., SnorEx.
Soft-palate lifters
Tongue trainers.
Mandibular repositioning or advancement devices (MRD / MAD)
Table 3. Types of Oral Devices

The American Academy of Sleep Medicine (AAOSM) recommends the use of oral appliances for those suffering from primary snoring or mild to moderate OSA. It can also be offered to those who have poor compliance to CPAP therapy or are intolerant to it, or those who are either not willing or unfit for surgery. ${ }^{21}$

These devices are worn only during sleeping hours. They reposition the tongue (anteriorly) or the mandible, thereby widening the upper airway. The exact mechanism of action is not yet known but they may either alter the shape of upper airway or augments its caliber. Another proposed mechanism of action suggests improvement in tone of muscles of upper airway, thereby decreasing their propensity to collapse.
Repositioning the tongue or mandible more anteriorly can widen the upper airway.

A tongue-retaining device is made of synthetic material which is applied to both upper and lower jaw. It consists of an anterior plastic bulb which holds the tongue in a forward position on applying gentle negative pressure, thereby widening the airway at the level of the oropharynx as well as the hypopharynx and preventing its prolapse during sleep.

Rapid maxillary expansion (RME), which is also known as rapid palatal expansion, is a process of widening the upper airway. Itis a skeletal type of expansion that involves use of oral appliance therapy (OAT) to separate the mid-palatal suture, resulting in maxillary shelves moving away from each other. The oral appliance applies heavy and rapid force on the posterior teeth, which exceeds the limit of orthodontic tooth movement and sutural resistance. This causes force to be transferred to the suture line as there is not enough time for tooth movement to occur which leads to opening up of the suture line while the teeth move only minimally relative to their supporting bone.

The indications of RME include patients with a transverse discrepancy $>4 \mathrm{~mm}$, with maxillary molars inclining towards buccal side (to compensate for the transverse skeletal discrepancy), cleft lip and palate patients with collapsed maxillae, maxillary protraction in class III treatment by disrupting the system of sutures, which connect the maxilla to the cranial base and moderate maxillary crowding to gain arch length.

The contraindications include patients, whose growth spurt has abated, have recession on the buccal aspect of the molars, anterior open bite, steep mandibular plane, convex profiles and who are non-compliant. There are several disadvantages of rapid palatal expanders including discomfort (because of heavy forces used and traumatic separation of the mid-palatal suture), rotated molars which are not amenable to correction, cooperative patient or parent to activate the appliance, open bite, recurrence, microtrauma of the temporomandibular joint and midpalatal suture, root resorption and tissue impingement.

Camacho et al. in their meta-analysis, reported that RME can cause $70 \%$ reduction in AHI in children aged 6 - 8 years, suffering from POSA, but the widening of midpalatal suture can continue in adolescence as well. ${ }^{22}$ Guilleminault et al. in their study on children who underwent RME combined with adenoidectomy, found that POSA was resolved satisfactorily in most children along with normalization of polysomnography. ${ }^{23}$ In view of insufficient evidence, RME is not recommended in patients with normally developed maxillary arches. Machado-Junior et al. in their review of 215 children (mean age of 6.7 years), to see the effectiveness of RME in resolving symptoms of POSA, found the mean AHI to decrease significantly after treatment with RME. With careful repositioning of the tongue, RME can increase the width of maxilla and oppose the lips properly, which can decrease mouth breathing.

Villa et al, in their study, reported that the effects of RME were persistent even after 2 years post treatment in children with both malocclusion and POSA. ${ }^{24}$ Whereas, Guilleminault et al. in a retrospective analysis, reported relapse over a period of more than 6 years after RME. They suggested the cause of recurrence to be skeletal relapse after RME treatment. There is a need for further studies as well as randomized controlled 
trials extending over longer periods, to better understand the benefits of treatment with RME in POSA and reasons of recurrence. Mandibular repositioning appliances (MRAs), although used in treatment of adults with OSA, is not currently recommended in children for lack of evidence. ${ }^{25}$

Mandibular advancement appliances (MAA) stimulate the activity of the genioglossus muscle, which protrudes the mandible and widens the oropharynx laterally, thereby decreasing the risk of airway collapse and promoting skeletal development. Nazarali et al. in their review, reported MAAs to cause short-term improvements in decreasing AHI, and hence, opined against the use of these devices in treatment of paediatric population. ${ }^{26}$ The compliance in wearing a nonfixed versus fixed MAA as well as degree of mandibular retrognathia could have influenced the results of the AHI scores in these studies. ${ }^{26}$ To summarize, these studies suffer from problems like small sample sizes, lack of randomized studies, and lack of control groups. Thus, further studies are required to address these issues and define the role of MAAs in the treatment of POSA.

To assess the eligibility of patient for OAT therapy, the oral examination should include a tool to assess risk of caries, periodontal examination, assessment of temporomandibular joint including the muscles of mastication, occlusal analysis, and parafunctional habits, and orthodontic evaluation, if required.

Dental malocclusion (21\%), TMJ pain (15\%), and TMJ dislocation $(<5 \%)$ are the side effects of MRDs. Other side effects include excessive salivation, tongue dryness, tooth pain, posterior open bite, and insomnia, limitation of degree of lateral jaw movements. The adverse effects with MRDs is found in 25 - $60 \%$ of patients, although most of them are often mild and can be resolved with adjustment of the device. ${ }^{27}$ There is a concern regarding development of degenerative disease in TMJ with MRD use, although there are no long term studies yet to shed a light on this issue. Patient should be advised to keep a follow up at 2 weeks after applying TRD, followed by 1 month, and every 6 months thereafter. ${ }^{28}$ The devices are kept in place by the remaining dentition and exert heavy forces on the teeth. The devices should be checked at every follow up for distortion or damage.

A follow up sleep study should be done to assess the benefit of OAT, in terms of reduction in the AHI. Therefore, dentists should be well versed not only with OAT but also its follow up testing. Positional training includes change in the sleeping posture to avoid supine position which can be done by simple manoeuvres like placing a tennis ball in the centre of the back or positioning the pillows such that they cannot roll on to their back.

Myofunctional therapy is another non-surgical approach, which works on the principle of strengthening the muscles of the tongue and Orofacial structures, to promote nasal breathing and lip seal, by teaching patients to reposition their muscles in a more appropriate position. ${ }^{29}$ It includes nasal breathing exercises, labial opposition, lip tone, and tongue position which should be performed three times a day. ${ }^{30} \mathrm{~A}$ meta-analysis by Camacho et al. reported that myofunctional therapy reduces AHI by $62 \%$ in children. ${ }^{31}$ So, it may be offered as a part of a more holistic strategy in the management of POSA and promote proper oropharyngeal development in children.

\section{CONCLUSIONS}

OSA is a chronic sleep disorder which is often under-diagnosed due to lack of awareness. The manifestations in children are different from those in adults. Timely identification helps to prevent the adverse consequences associated with it. Dentists are often the first to recognise the symptoms of SDB in children and therefore, can play a crucial role in its early identification. For instance, a child may fall asleep while waiting in the lounge or in the dental chair which may be associated with snoring.

Also, the dentist may notice the presence of risk factors like enlarged tonsils, macroglossia, cranio-facial features and obesity. If the presence of symptoms or signs raise the suspicion of SDB, the dentist must further probe the symptoms in detail and counsel the parents regarding the need for further evaluation, if required.

Polysomnography remains the gold standard for the diagnosis of SDB as well as for monitoring the effects of different therapeutic approaches. The management of OSA includes non-invasive and invasive approaches. The noninvasive strategies include positive airway pressure, oral appliances, positional therapy, behavioural therapy, and myofunctional therapy. Although continuous positive airway pressure therapy is the most effective therapy for OSA, other strategies particularly, oral appliances offer an alternative for those with mild to moderate POSA, who are either not tolerating CPAP or refusing it.

The role of dentists has expanded from mere recognition of disorder to active management with the help of oral appliances. This is especially important in children where compliance with CPAP therapy may be of concern. Although treatment with OAT is not entirely devoid of side effects, they are relatively milder and can easily be dealt with. Although long term results of therapy with oral appliances are not yet known, the future appears quite promising. The current education curriculum may not be adequate in terms of providing proper training in identification as well as management of OSA. So, dentists should take the initiative to educate themselves about the various aspects of this disorder.

Financial or other competing interests: None.

Disclosure forms provided by the authors are available with the full text of this article at jemds.com.

\section{REFERENCES}

[1] Chan J, Edman JC, Koltai PJ. Obstructive sleep apnoea in children. Am Fam Physician 2004;69(5):1147-54.

[2] The report of an American Academy of sleep medicine task force. Sleep-related breathing disorders in adults: recommendations for syndrome definition and measurement techniques in clinical research. Sleep 1999;22(5):667-89.

[3] American Academy of Paediatric Dentistry. Policy on obstructive sleep apnoea. Pediatr Dent 2017;38(Special Issue):87-9.

[4] Guilleminault C, Sullivan SS. Towards restoration of continuous nasal breathing as the ultimate treatment goal in paediatric obstructive sleep apnoea. Enliven: Paediatrics and Neonatol Biology 2014;1(1):1-5. 
[5] Biggs SN, Walter LM, Jackman AR, et al. Long-term cognitive and behavioral outcomes following resolution of sleep disordered breathing in preschool children. PLoS One 2015;10(9):e0139142.

[6] Marcus CL, Brooks LJ, Draper KA, et al. Diagnosis and management of childhood obstructive sleep apnoea syndrome. Paediatrics 2012;130(3):714-55.

[7] Redline S, Tishler PV, Schluchter M, et al. Risk factors for sleep-disordered breathing in children: associations with obesity, race and respiratory problems. Am J Respir Crit Care Med 1999;159(5 Pt 1):1527-32.

[8] Marcus CL. Sleep disordered breathing in children. Am J Respir Crit Care Med 2001;164(1):16-30.

[9] Capua M, Ahmadi N, Shapiro C. Overview of obstructive sleep apnoea in children: exploring the role of dentists in diagnosis and treatment. J Can Dent Assoc 2009;75(4):285-9.

[10] Ronen O, Malhotra A, Pillar G. Influence of gender and age on upper-airway length during development. Paediatrics 2007;120(4):e1028-34.

[11] Naimark A, Cherniack RM. Compliance of the respiratory system and its components in health and obesity. J Appl Physiol 1960;15(3):377-82.

[12] Bhattacharjee R, Kim J, Kheirandish-Gozal L, et al. Obesity and obstructive sleep apnoea syndrome in children: a tale of inflammatory cascades. Pediatr Pulmonol 2011;46(4):313-23.

[13] Schellenberg JB, Maislin G, Schwab RJ. Physical findings and the risk for obstructive sleep apnoea: the importance of oropharyngeal structures. Am J Respir Crit Care Med 2000;162(2 Pt 1):740-8.

[14] Lobstein T, Jackson-Leach R. Child overweight and obesity in the USA: prevalence rates according to IOTF definitions. Int J Pediatr Obes 2007;2(1):62-4.

[15] Dayyat E, Kheirandish-Gozal L, Capdevila OS, et al. Obstructive sleep apnoea in children: relative contributions of body mass index and adenotonsillar hypertrophy. Chest 2009;136(1):137-44.

[16] Guilleminault C, Abad VC, Chiu HY, et al. Missing teeth and paediatric obstructive sleep apnoea. Sleep Breath 2016;20(2):561-8.

[17] DiFrancesco RC, Junqueira PAS, Trezza PM, et al. Improvement of bruxism after T \& A surgery. Int J Pediatr Otorhinolaryngol 2004;68(4):441-5.

[18] Bucks RS, Olaithe M, Eastwood P. Neurocognitive function in obstructive sleep apnoea: a meta-review. Respirology 2013;18(1):61-70.
[19] Vorona RD, Ware JC. Sleep disordered breathing and driving risk. Curr Opin Pulm Med 2002;8(6):506-10.

[20]Johns MW. A new method for measuring daytime sleepiness: the epworth sleepiness scale. Sleep 1991;14(6):540-5.

[21] Practice parameters for the treatment of snoring and obstructive sleep apnoea with oral appliances. American Sleep Disorders Association. Sleep 1995;18(6):511-3.

[22] Camacho M, Chang ET, Song SA, et al. Rapid maxillary expansion for paediatric obstructive sleep apnoea: a systematic review and meta-analysis. Laryngoscope 2017;127(7):1712-9.

[23] Guilleminault C, Monteyrol PJ, Huynh NT, et al. Adenotonsillectomy and rapid maxillary distraction in prepubertal children, a pilot study. Sleep Breath 2011;15(2):173-7.

[24] Villa MP, Rizzoli A, Miano S, et al. Efficacy of rapid maxillary expansion in children with obstructive sleep apnoea syndrome: 36 months of follow-up. Sleep Breath 2011;15(2):179-84.

[25] Barewal RM, Hagen CC. Management of snoring and obstructive sleep apnoea with mandibular repositioning appliances: a prosthodontics approach. Dent Clin North Am 2014;58(1):159-80.

[26] Nazarali N, Altalibi M, Nazarali S, et al. Mandibular advancement appliances for the treatment of paediatric obstructive sleep apnoea: a systematic review. Eur J Orthod 2015;37(6):618-26.

[27] Schmidt-Nowara W, Lowe A, Wiegand L, et al. Oral appliances for the treatment of snoring and obstructive sleep apnoea: a review. Sleep 1995;18(6):501-10.

[28] Ivanhoe JR. Treatment of upper airway sleep disorder patients with dental devices. Clinical Maxillofacial Prosthetics. Chicago: Quintessence 2000:215-31.

[29] Guilleminault C, Huang YS, Monteyrol PJ, et al. Critical role of myofascial reeducation in paediatric sleep-disordered breathing. Sleep Med 2013;14(6):518-25.

[30] Frey L, Green S, Fabbie P, et al. The essential role of the com in the management of sleep-disordered breathing: a literature review and discussion. Int J Orofacial Myology 2014;40:42-55.

[31] Camacho M, Certal V, Abdullatif J, et al. Myofunctional therapy to treat obstructive sleep apnoea: a systematic review and meta-analysis. Sleep 2015;38(5):669-75. 